\title{
Pediatric Tuina For Functional Constipation In Children: Study Protocol For A Randomized Controlled Trail
}

\section{Xinghe Zhang}

Yunnan University of Chinese Medicine

Luan $\mathrm{Hu}$

Yunnan Province Hospital of TCM

Li Li

Yunnan University of Chinese Medicine

Yuanwang Wang

Yunnan University of Chinese Medicine

\section{Can Zhang}

Yunnan University of Chinese Medicine

\section{Jinyan Su}

Kunming Children's Hospital

Hua Di

Zhejiang Provincial People's Hospital

\section{Qing Gao}

Shandong Province Hospital of TCM

Xiantao Tai ( $\nabla$ taixiantao@163.com )

Yunnan University of Chinese Medicine

\section{Taipin Guo}

Yunnan University of Chinese Medicine

\section{Research Article}

Keywords: pediatric Tuina, functional constipation, randomized controlled trail

Posted Date: November 10th, 2021

DOl: https://doi.org/10.21203/rs.3.rs-1057133/v1

License: (c) (i) This work is licensed under a Creative Commons Attribution 4.0 International License. Read Full License 


\section{Abstract}

Background: Functional constipation (FC) is a common functional gastrointestinal disorder (FGID), which brings many negative impacts to the children's daily life. Pediatric Tuina has been proved to be a potential therapy for functional constipation (FC). However, the evidence for its effectiveness and safety is insufficient due to the lack of high-quality study. This study aims to evaluate the efficacy and safety of pediatric Tuina for children with FC.

Methods/design: This study is a randomized, controlled, multicentre, clinical trial. We will include 176 children with FC from five hospitals. The participants will be randomly allocated into two groups: the pediatric Tuina group and the Medilac-Vita group. This study will include a 1-week actual treatment period and a 2-week follow-up period. Primary outcomes are weekly spontaneous bowel movements (SBMs), weekly complete spontaneous bowel movements (CSBMs). The secondary outcomes are effective rate, stool form, distress sensation, and glycerine enema rate. The assessment will be performed each week. Adverse event will be monitored in treatment period and follow-up period.

Discussion: This study is designed to evaluate the efficacy and safety of pediatric Tuina for children with FC, and we hypothesize that pediatric Tuina is more effective than probiotics. It will provide reliable evidence and support for the treatment of FC by pediatric Tuina.

Trail registration: This protocol was registered in Chinese Clinical Trial Registry (ChiCTR2100046485).

\section{Background}

Functional constipation (FC), a common functional gastrointestinal disorder (FGID), which is characterized by infrequent, difficult and incomplete defection [1,2]. According to the survey report, the prevalence of FC in children ranges from $0.7-29.6 \%$ (median $12 \%$ )[3-5]. Although FC is not life-threatening, it significantly influences the patients' quality of life and consumes many medical resources[6-8]. Currently, there are two broad categories of treatments for FC: pharmacological and nonpharmacological [9]. Pharmacological treatments have limited therapeutic effects but considerable side effects, while the symptom tends to recur[10-12]. Nonpharmacological treatments, such as physical activity, fiber and fluid intake, are recommended to regulate bowel movement, whereas the evidence is still insufficient and strength of recommendation is weak $[9,13,14]$. Therefore, it is urgently to find an effective and safe therapy for FC in children.

Pediatric Tuina is a special massage therapy based on traditional Chinese medicine (TCM), which can be traced back to Qin Dynasty (220 B.C.) and the complete system was established in the Ming Dynasty (1601 A.D.) [15]. Up to now, many universities of TCM offer the subjects of pediatric Tuina for medicos[16]. The special meridianacupoint theory is different from adults, most acupoints are located on finger, palm, forearm, while few acupoints located on head, abdomen and back[17]. The manipulations such as rubbing, pushing, pinching, kneading, vibrating, are characterized by light, fast, gentle[18].

In recent years, many randomized controlled trials (RCTs) have indicated that pediatric Tuina may be an effective treatment for FC in children[19-23]. However, the inadequacy of the study design restricts the quality and credibility [24-27]. Thus, we designed a randomized controlled trial to evaluate the efficacy and safety of pediatric Tuina for children with FC. Meanwhile, we aim to compared the effect of pediatric Tuina with Medilac-Vita.

\section{Methods/design}




\section{Study design}

This study is a prospective, randomized, multicentre, controlled design, and it will be conducted from December 2021 to December 2024. We will recruit 176 participates from 5 hospitals in 3 different provinces (Yunnan province, Zhejiang province, and Shandong Province). The eligible participants will be randomly allocated to 2 groups in a 1:1 ratio. The randomization sequence will be computer generated by the Clinical Research Center of Yunnan University of Chinese Medicine. Every participant will receive a 1-week treatment period and a 2-week follow-up. Basic information and outcomes will be assessed at baseline and after each week. The flowchart is shown in Fig. 1 and the schedule is shown in Table 1.

Table 1 Schedule of the study

\begin{tabular}{|c|c|c|c|c|c|c|c|c|c|c|c|c|c|c|}
\hline \multirow{2}{*}{ Item } & \multicolumn{14}{|c|}{ Days } \\
\hline & -7 & - & 0 & 1 & 2 & 3 & 4 & 5 & 6 & 7 & - & 14 & - & 21 \\
\hline Eligibility screen & $\times$ & & & & & & & & & & & & & \\
\hline Informed consent & $\times$ & & & & & & & & & & & & & \\
\hline Baseline & $\times$ & $x$ & $x$ & & & & & & & & & & & \\
\hline Allocation & & & $\times$ & & & & & & & & & & & \\
\hline Pediatric Tuina & & & $\times$ & & $\times$ & & $\times$ & & $x$ & & & & & \\
\hline Medilac-Vita & & & $\mathbf{x}$ & $\mathbf{x}$ & $x$ & $x$ & $\mathbf{x}$ & $\mathbf{x}$ & $x$ & & & & & \\
\hline Follow-up & & & & & & & & & & $x$ & $\times$ & $x$ & $\mathbf{x}$ & $x$ \\
\hline \multirow[t]{2}{*}{ Stool dairy } & $\mathbf{x}$ & $\times$ & $\mathbf{x}$ & $\mathbf{x}$ & $\mathbf{x}$ & $x$ & $\mathbf{x}$ & $\mathbf{x}$ & $\mathbf{x}$ & $x$ & $x$ & $x$ & $x$ & $x$ \\
\hline & \multicolumn{14}{|c|}{ Assessment } \\
\hline SBMs & & & $\mathbf{x}$ & & & & & & & $\times$ & & $x$ & & $\mathbf{x}$ \\
\hline CSBMs & & & $\times$ & & & & & & & $\times$ & & $\times$ & & $\times$ \\
\hline Effective rate & & & $x$ & & & & & & & $x$ & & $x$ & & $x$ \\
\hline m-BSFS & & & $\times$ & & & & & & & $\times$ & & $x$ & & $\times$ \\
\hline Distress sensation & & & $\times$ & & & & & & & $\times$ & & $x$ & & $x$ \\
\hline \multirow[t]{2}{*}{ Glycerine enema rate } & & & $\times$ & & & & & & & $\times$ & & $x$ & & $\times$ \\
\hline & \multicolumn{14}{|c|}{ Participants safety } \\
\hline Adverse events & & & $\mathbf{x}$ & $\mathbf{x}$ & $\mathbf{x}$ & $\times$ & $\mathbf{x}$ & $\mathbf{x}$ & $\mathbf{x}$ & $\times$ & $\mathbf{x}$ & $x$ & $\mathbf{x}$ & $x$ \\
\hline
\end{tabular}

This trial is reported in line with the Standard Protocol Items Recommendations for Interventional Trials (SPIRIT) guidelines, and details are provided in Additional file 1. This trial follows the principles of the Declaration of Helsinki (Version Edinburgh 2000). This protocol was registered in Chinese Clinical Trial Registry (ChiCTR2100046485), and approved by the Hospital Ethics Committee of Second Affiliated Hospital of Yunnan University of Chinese Medicine (2021-014).

\section{Participants}

This study will include 176 participants from the departments of Acupuncture and Tuina in Second Affiliated Hospital of Yunnan University of Chinese Medicine, Yunnan Province Hospital of TCM, Kunming Children's Hospital, Zhejiang Provincial People's Hospital, and Shandong Province Hospital of TCM. The participants will be appropriately compensated after they complete the entire procedure. However, parents or legal guardians of the participants have the right to withdraw from this study at any time, for any reason. And they will not face any discrimination or hardship in the hospital. The participants will be the children with FC and they must meet the following eligibility criteria. 


\section{Inclusion criteria}

1. Children aged 1-7years.

2. Children who meet the diagnostic criteria for functional constipation (Rome IV)[28, 29]. Must include 1 month of at least 2 of the following in infants up to 4 years of age: (1) 2 or fewer defecations per week; (2) History of excessive stool retention; (3) History of painful or hard bowel movements; (4) History of large-diameter stools; (5) Presence of a large fecal mass in the rectum. In toilet-trained children, the following additional criteria may be used: (6) At least 1 episode/week of incontinence after the acquisition of toileting skills; (7) History of large-diameter stools that may obstruct the toilet. For children over 4 years of age, there must include 2 or more of the following occurring at least once per week for a minimum of 1 month with insufficient criteria for a diagnosis of irritable bowel syndrome: (1) 2 or fewer defecations in the toilet per week in a child of a developmental age of at least 4 years; (2) At least 1 episode of fecal incontinence per week; (3) History of retentive posturing or excessive volitional stool retention; (4) History of painful or hard bowel movements; (5) Presence of a large fecal mass in the rectum; (6) History of large diameter stools that can obstruct the toilet.

3. Parents or legal guardians are motivated to participate in the trial.

4. Parents or legal guardians sign written informed consent.

\section{Exclusion criteria}

1. A history of intestinal surgery.

2. Receiving gastrointestinal prokinetic agent or laxative treatments 14 days before enrollment.

3. Receiving pediatric Tuina or probiotics 14 days before enrollment.

4. Children with Hirschsprung's disease.

5. Children with neurological and psychiatric disorders (e.g., cerebral palsy, autism, spina bifida, anorexia nervosa).

6. Children with any organic or metabolic diseases of hematopoietic, endocrine, immune systems.

\section{Randomization and blinding}

Eligible FC children will be randomly assigned into two groups at 1:1 ratio. The randomization will be conducted by using a computerized random number generator, the Clinical Research Center of Yunnan University of Chinese Medicine. There will be opaque envelopes with random numbers for random allocation. The envelopes will be managed by an independent member who not involved in the treatment procedure or data analysis. The opaque envelope will not be opened until the participant is about to receive treatment, and then the participant will be grouped.

There are two different types of interventions in this study. It is impossible to blind therapists and children's parents or legal guardians. Nonetheless, outcome assessors and the data analyst will be blinded to the procedure and results of randomization, group allocation and intervention.

\section{Interventions}

To ensure all manipulations follow the standard procedures. The therapists in this trial own official medical licence and hold master's degree in pediatric Tuina. During this period, participants are not allowed to use any other interventions, at least in principle. If participants take any other interventions in this period, they will be defined as non-adherent and will be dealt with drop out. 


\section{Pediatric Tuina group}

Participants will receive pediatric Tuina treatment for 20 min each session. Once two days for four times. Treatment will be performed with the child lies in bed, with the placate by parents or legal guardians at bedside. The standard pediatric Tuina includes 7 acupoints, which are determined by the literature review[26] and the pediatric Tuina professor with 30 years' experience. Manipulation times is depended on the children's age. Detailed information of acupoints, manipulation methods and times is provided in Table 2. The location of acupoints and manipulations are shown in Additional file2. The manipulations are light, fast, and gentle.

Table 2

Acupoints, manipulation methods and times

\begin{tabular}{|c|c|c|c|c|c|c|c|c|}
\hline \multirow[t]{2}{*}{ Age } & \multicolumn{8}{|l|}{ Times } \\
\hline & $\begin{array}{l}\text { Large } \\
\text { intestine } \\
\text { meridian, } \\
\text { reducing, } \\
\text { pushing }\end{array}$ & $\begin{array}{l}\text { Lung } \\
\text { meridian, } \\
\text { reducing, } \\
\text { pushing }\end{array}$ & $\begin{array}{l}\text { Liufu, } \\
\text { reducing, } \\
\text { pushing }\end{array}$ & $\begin{array}{l}\text { Sanguan, } \\
\text { reinforcing, } \\
\text { pushing }\end{array}$ & $\begin{array}{l}\text { Abdomen, } \\
\text { clockwise, } \\
\text { rubbing \& } \\
\text { vibration }\end{array}$ & $\begin{array}{l}\text { Tianshu, } \\
\text { vertical, } \\
\text { pressing } \\
\& \\
\text { vibration }\end{array}$ & $\begin{array}{l}\text { Qijiegu, } \\
\text { downward, } \\
\text { pushing }\end{array}$ & $\begin{array}{l}\begin{array}{l}\text { Guiwei, } \\
\text { clockwise, }\end{array} \\
\text { rubbing }\end{array}$ \\
\hline $\begin{array}{l}\geq 1 \\
\text { years } \\
\text { old } \\
\text { and } \\
<4 \\
\text { years } \\
\text { old }\end{array}$ & 300 & 200 & 200 & 20 & 300 & 300 & 300 & 200 \\
\hline $\begin{array}{l}\geq 4 \\
\text { years } \\
\text { old } \\
\text { and } \\
<7 \\
\text { years } \\
\text { old }\end{array}$ & 500 & 400 & 400 & 40 & 500 & 500 & 500 & 400 \\
\hline
\end{tabular}

\section{Medilac-Vita group}

According to the guideline of the Chinese Preventive Medicine Association (CPMA) in 2017, probiotics such as Medilac-Vita are recommended for the treatment of FC in children[30]. Participants assigned to this group will receive probiotics treatment. Medilac-Vita (combined bacillus subtilis and enterococcus faecium granules with multivitamines, live), which has been widely used for FC in children[31-34]. Participants will take $1 \mathrm{~g}$ (1 to 4 years old) or $2 \mathrm{~g}$ (4 to 7 years old) Medilac-Vita (Hanmi Pharm Co., Ltd, Beijing, China) dissolved in $100 \mathrm{ml}$ water $\left(40^{\circ} \mathrm{C}\right.$ ), according to the drug instruction (Additional file 3). Twice a day, 7 consecutive days in total. After treatment period, parents or guardians of the participants will be required to return any untaken drugs to avoid adverse consequences for follow-up.

\section{Outcomes measurement}

A printed or electronic case report form (CRF) with the random number for each participant will be used to record all information. Three assessors who are blind to the group allocations will collect the basic information and assess 
the outcomes every week from the baseline to the end of follow-up. The first one will assess outcomes from Second Affiliated Hospital of Yunnan University of Chinese Medicine, Yunnan Province Hospital of TCM, and Kunming Children's Hospital. The second one is for Zhejiang Provincial People's Hospital. The third one is for Shandong Province Hospital of TCM.

\section{Stool diary}

Parents or legal guardians are required to keep a stool diary from baseline to intervention period and follow-up period. The stool diary contains 5 domains: weekly spontaneous bowel movements (SBMs), weekly complete spontaneous bowel movements (CSBMs), stool form, distress sensation, and glycerine enema rate.

\section{Primary outcomes}

The primary outcome are weekly SBMs and/or CSBMs. Children older than 4 years old could answer whether defecation is complete. The assessors will check the stool diary and gather the data from baseline until the end of follow-up.

\section{Secondary outcomes}

The secondary outcomes are effective rate, stool form, distress sensation, and glycerine enema rate. Effective rate is the number of participants who are effective (on average, at least one bowel movement in three days, stool softening, defecate unobstructed) divided by total number of the group. Stool form will be measured according to the modified Bristol stool form scale (m-BSFS) (Table 3), which classifies the form of children feces into 5 types from separate hard lumps (type 1) to entirely watery (type 5) [35, 36]. Distress sensation measured in the range of 0-3 represents the intensity of distress during defecation, " 0 " means no cry or grimace, " 1 " means slight cry and grimace, "2" means medium cry and grimace, and " 3 " means hysterical cry and grimace. Glycerine enema rate is the times of using glycerine enema divided by the times of defecation, within a week.

Table 3 Modified Bristol stool form

\begin{tabular}{|c|c|c|c|c|c|}
\hline Type & 1 & 2 & 3 & 4 & 5 \\
\hline Sample & 0 & 0 & 0 & & \\
\hline Character & Separate hard lumps & $\begin{array}{c}\text { Sausage-shaped but } \\
\text { lumpy }\end{array}$ & $\begin{array}{c}\text { Like sausage or } \\
\text { snake, smooth and } \\
\text { soft }\end{array}$ & $\begin{array}{c}\text { Fluffy, ragged edges, } \\
\text { mushy stool }\end{array}$ & $\begin{array}{c}\text { Waterly, no solid } \\
\text { pieces }\end{array}$ \\
\hline Score & 1 & 2 & 3 & 4 & 5 \\
\hline
\end{tabular}

\section{Safety assessment and dispose}

The type, time of occurrence, severity, duration of the adverse events will be recorded in detail from day 0 to 28 . If emesis, severe diarrhea, dehydration, skin injury, fainting, infection, or other severe adverse events occur, the participants should be discontinued immediately from treatment and given appropriate treatment directly at the located hospital. And then, the detail adverse event will be reported to the Ethics Committee. 


\section{Adherence improvement}

Parents will be asked to hand over the remaining probiotics after the drop out or at the end of the full treatment. After the follow-up period, parents will be given three free pediatric Tuina sessions or two courses of probiotics.

\section{Sample size}

According to a previous relevant study that compared the effective rate of pediatric Tuina (92.5\%) and Medilac-Vita (72.5\%)[23]. We anticipate an effective rate of $90 \%$ in the pediatric Tuina group, $70 \%$ in the Medilac-Vita group. Considering $a=0.05,1-\beta=0.90, p_{1}=0.90, p_{2}=0.70, u_{0.05 / 2}=1.96, u_{0.1}=1.282$ [37],

$n=1641.4 \times\left[\frac{(1.96+1.282)}{\sin ^{-1} \sqrt{0.90}-\sin _{-1}^{0.70}}\right]^{2}=79.02 \approx 79$. With a drop-out of $10 \%, 88 \mathrm{FC}$ children will be finally recruited in each group. Totally 176 participants will be finally recruited in this study.

\section{Data collection}

In this study, an independent data administrator will collect data every three months, from three assessors. The data of each participants including basic information and outcomes. The basic information including participant's gender, age, height, weight, history of pregnancy and delivery, past medical history, present condition, phone and WeChat number of parents or legal guardians. Outcomes will be collected four times from baseline to the end of follow-up.

\section{Data management}

The CRFs completed in 5 hospitals will be sent to the research office based in the School of Second Clinical Medicine, Yunnan University of Chinese Medicine. Two team members who are blind to the group allocations will perform double-data entry and summarize the data into electronic dataset. Meanwhile, the data administrator will monitor and check for errors. The data administrator and statistician will be blind to the group allocations, and have the right to access the final dataset.

\section{Statistical analysis}

The statistician will perform statistical analysis by using SPSS 19.0 statistics software (IBM Co., Armonk, NY, USA) and GraphPad 7.04 statistics software (GraphPad Software Co., San Diego, California, USA). Continuous variables are presented as the mean and standard deviation (SD). While the nonnormally distributed variables are presented as the median and interquartile range (IQR). Categorical variables are described as numbers and percentages (\%).

Before the comparison, the normality and homogeneity of variance will be tested. Normality of continuous variables (SBMs, CSBMs, m-BSFS, distress sensation, and glycerine enema rate) will be determined by Shapiro-Wilk test, Kolmogorov-Smirnov test, Skewness and Kurtosis. Homogeneity of variance of continuous variables will be determined by $F$ test, Brown-Forsythe and Bartlett's test. Independent-Sample $t$-test or nonparametric test (MannWhitney test) will be used when compared between two groups. Paired $t$-test or nonparametric test (Wilcoxon test) will be performed to compare within a group. For the categorical variables (effective rate), the $\chi 2$ test will be performed to evaluate the significance of the difference. The statistical analysis will be 2-sided test with a significance level of 0.05 .

The missing data were will be deal with expectation maximization, last observation carried forward method or listwise deletion. Subgroup analysis will be conducted according to the subject's age ( $<4$ years and $\geq 4$ years), which depend on the normality and homogeneity of variance of the data. 


\section{Discussion}

Pediatric Tuina is a Chinese massage therapy based on the special meridian-acupoint theory of TCM. Due to the characteristics of pediatric Tuina, all manipulations are performed on the body surface. Under some circumstances, cloaks and other covering may be used to blind parents or guardians[38]. However, most parents hope that child's symptoms get a quick relief, despite FC is not life-threatening. In case of the efficacy (sham Tuina) cannot be guaranteed, it easily lead to doctor-patient disputes. Therefore, this study set probiotics with definite efficacy as the control.

Sample size calculation should be based on the results of relevant high-quality studies by principle. Limited by the methodological flaws of the existing studies, there were no objective indicators (e.g., SBM, CSBM, BSFS, m-BSFS) as outcomes. The effective rate was most widely used in pediatric Tuina for FC. Therefore, the sample size of this study set effective rate as reference. However, this study also set the defecation frequency, stool form, and distress sensation those directly related to constipation as the outcomes. Under the circumstance that lumpy stool and difficult defecation, glycerine enema is usually used to assist defecate. Anal injection of glycerine enema can lubricate the colon to promote the excretion of stool. Therefore, glycerine enema rate can indicate whether difficult defecation and whether child can complete defecate on his own. Other evaluation indicators such as Cleveland Constipation Score (CCS), Patient Assessment of Constipation Symptom (PAC-SYM), Patient Assessment of Constipation Quality of Life Questionnaire (PAC-QoL) are not suitable for children with FC, because children (especially toddler) could not express their feelings directly and accurately[39-42].

As a complementary and alternative therapy, standardization and quantification of massage manipulations have always been stumbling blocks which limit the scientific research and development of massage. The complex manipulations of massage determine that the robot can not completely replace the therapists. In china, pediatric Tuina is a medical treatment rather than a nursing skill. Although all manipulations will be performed by physicians with a master's degree in pediatric Tuina, differences in manipulations may still exist between different physicians. In order to solve the differences, we will conduct unified training of pediatric Tuina before the study started. Electronic test glove which can measure pressure, amplitude, frequency, angular velocity will be used for examine the therapist's manipulation (Fig. 2 and Additional file 3).

In conclusion, this study is the first to investigate the differences in defecation frequency and stool form between pediatric Tuina and probiotics for children with FC. It provides a potential therapy for the treatment of FC in children, and certify the efficacy, safety, and advantage of pediatric Tuina.

\section{Trial status}

This trial was registered on 16 May 2021. The trial is currently in the stage of unified training of pediatric Tuina.

\section{Abbreviations}

CPMA: Chinese Preventive Medicine Association; CRF: Case report form; CSBMs: Complete spontaneous bowel movements; FC: Functional constipation; FGID: Functional gastrointestinal disorder; IQR: Interquartile range; mBSFS: Modified Bristol stool form scale; RCTs: Randomized controlled trials; SBMs: Spontaneous bowel movements; SD: Standard deviation; SPIRIT: Standard Protocol Items Recommendations for Interventional Trials; TCM: Traditional Chinese Medicine. 


\section{Declarations}

\section{Acknowledgments}

Not applicable

\section{Authors' contributions}

XHZ, LH and LL are responsible for the design and drafting of the manuscript. YYW, CZ, JYS, HD, QG will participate in the implementation of the study. XTT, TPG provided the funding, and total design. The authors above contributed to the revision and approved the final manuscript.

\section{Funding}

This study is funded by the Special Subsidy of Yunnan Province (2020009) from People's Government of Yunnan Province, Youth Top Talent Project of Ten-thousand Talent Plan (YNWR-QNBJ-2019-257) from the Human Resources \& Social Security Department of Yunnan Province. The funding bodies do not exert any role in the design; collection, management, analysis, and interpretation of data; writing of the report; and the decision to submit the report for publication.

\section{Availability of data and materials}

No data were used to support this protocol. The outcomes data of the trial will be published within the final study manuscript.

\section{Ethics approval and consent to participate}

This trial has been approved by the Ethics Committee of the Second Affiliated Hospital of Yunnan University of Chinese Medicine (Approved number: 2012-014). All parents or legal guardians of enrolled participants will be informed and sign a written consent form.

\section{Consent for publication}

All authors read and approved the final manuscript.

\section{Competing interests}

The authors declare that they have no competing interests.

\section{References}

1.Mearin F, Lacy BE, Chang L, Chey WD, Lembo AJ, Simren M, et al. Bowel Disorders. Gastroenterology. 2016;150(6):1393-407.

2.Drossman DA. The functional gastrointestinal disorders and the Rome III process. Gastroenterology. 2006;130(5):1377-90.

3.Mugie SM, Benninga MA, Di Lorenzo C. Epidemiology of constipation in children and adults: a systematic review. Best Pract Res Clin Gastroenterol. 2011;25(1):3-18. 
4.Rajindrajith S, Devanarayana NM. Constipation in children: novel insight into epidemiology, pathophysiology and management. J Neurogastroenterol Motil. 2011;17(1):35-47.

5.Van den Berg MM, Benninga MA, Di Lorenzo C. Epidemiology of childhood constipation: a systematic review. Am J Gastroenterol. 2006;101(10):2401-9.

6.Ansari H, Ansari Z, Lim T, Hutson JM, Southwell BR. Factors relating to hospitalisation and economic burden of paediatric constipation in the state of Victoria, Australia, 2002-2009. J Paediatr Child Health. 2014;50(12):993-9.

7.Guerin A, Carson RT, Lewis B, Yin D, Kaminsky M, Wu E. The economic burden of treatment failure amongst patients with irritable bowel syndrome with constipation or chronic constipation: a retrospective analysis of a Medicaid population. J Med Econ. 2014;17(8):577-86.

8.Wald A, Sigurdsson L. Quality of life in children and adults with constipation. Best Pract Res Clin Gastroenterol. 2011;25(1):19-27.

9. Tabbers MM, DiLorenzo C, Berger MY, Faure C, Langendam MW, Nurko S, et al. Evaluation and treatment of functional constipation in infants and children: evidence-based recommendations from ESPGHAN and NASPGHAN. J Pediatr Gastroenterol Nutr. 2014;58(2):258-74.

10.Gordon M, Naidoo K, Akobeng AK, Thomas AG. Osmotic and stimulant laxatives for the management of childhood constipation. Cochrane Database Syst Rev. 2012(7):CD009118.

11.Ford AC, Suares NC. Effect of laxatives and pharmacological therapies in chronic idiopathic constipation: systematic review and meta-analysis. Gut. 2011;60(2):209-18.

12.Tabbers MM, Boluyt N, Berger MY, Benninga MA. Clinical practice : diagnosis and treatment of functional constipation. Eur J Pediatr. 2011;170(8):955-63.

13.Mearin F, Ciriza C, Minguez M, Rey E, Mascort JJ, Pena E, et al. Clinical Practice Guideline: Irritable bowel syndrome with constipation and functional constipation in the adult. Rev Esp Enferm Dig. 2016;108(6):332-63.

14.Shin JE, Jung HK, Lee TH, Jo Y, Lee H, Song KH, et al. Guidelines for the Diagnosis and Treatment of Chronic Functional Constipation in Korea, 2015 Revised Edition. J Neurogastroenterol Motil. 2016;22(3):383-411.

15.Li YN, Yang PL, Wu JY. The development history of pediatric Tuina. Beijing J TCM. 2009;28(2):142-4. [In Chinese].

16.Chu X, Lu D. A brief discussion on the present situation and reform ideas of pediatric Tuina in higher Chinese Medicine education. J Changsha Med Col. 2016;14(3):58-9. [In Chinese].

17.Ma SJ. The development of human body channels and collaterals was discussed by Tuina point of children. Jiangsu J TCM. 2014;46(3):11-3. [In Chinese].

18.Tai XT, Xiong L. Pediatric Tuina. Beijing: China Press of Traditional Chinese Medicine; 2011.[In Chinese].

19.Cai YH, Huang XX, Zhang HM, Fu DD, Xu L. Clinical observation on the treatment of 65 cases of functional constipation with transspleen - induced stagnation Tuina in children. Zhejiang J TCM. 2020;55(04):276. [In Chinese]. 
20.Han SC, He S. Tuina for 200 cases of constipation in children. Chin J Conval Med. 2013;22(04):344-5. [In Chinese].

21.Jia GH. Observation on 36 cases of children functional constipation treated with pediatric Tuina. Hunan J TCM. 2018;34(07):123-4. [In Chinese].

22.Wang GY, Li YL, Pu ZX. Observation of therapeutic effect of pediatric Tuina combined with probiotics on functional constipation in children. J Military Surgeon in Southwest China. 2013;15(02):123-5. [In Chinese].

23.Zhou XQ, Hu SP, Li YX. Clinical observation of 40 cases of functional constipation in children treated by pediatric Tuina. J Pediatrics of TCM. 2020;16(04):93-5. [In Chinese].

24.Chen YP, Zhao H, Lin YP. Meta-analysis of clinical efficacy of Tuina in the treatment of constipation in children Guangming J TCM. 2018;33(24):3764-7. [In Chinese ].

25.Hu AN, Gao PY, Wu F, Liu DM. Clinical progress of pediatric Tuina in the treatment of functional constipation in children Chin Mani \& Reha Med. 2019;10(03):57-60. [In Chinese].

26.Huang MY, Li YC, W.L. L, Huang H, Wu QW, Lin LL. Literature review on the treatment of children constipation by pediatric Tuina. Fujian J TCM. 2020;51(02):67-8. [In Chinese].

27.Liu NF, Guo SJ, Feng JH. Research progress of Tuina therapy for functional constipation in children. Med Recapitulate. 2017;23(19):3907-11. [In Chinese].

28.Benninga MA, Faure C, Hyman PE, St James Roberts I, Schechter NL, Nurko S. Childhood Functional Gastrointestinal Disorders: Neonate/Toddler. Gastroenterology. 2016;150(6):1443-55.

29.Hyams JS, Di Lorenzo C, Saps M, Shulman RJ, Staiano A, Rasquin A, et al. Childhood functional gastrointestinal disorders: child/adolescent. Gastroenterology. 2016;150(6):1456-68.

30.Chen J, Cheng Q, Huang Y, Huang YK. Evidence-based guidelines for the clinical application of probiotics in pediatric medicine. Chin J Prac Pedia. 2017;32(2):81-90. [In Chinese].

31.Chen K, Cheng XR, Zhang L, Luo HY, Gao N, Wang J, et al. Clinical efficacy evaluation of combined bacillus subtilis and enterococcus faecium granules with multivitamines (live) in improving functional constipation in children. Chin J Prac Pedia. 2013;28(08):618-20. [In Chinese].

32.Hu D. Medilac-Vita to treat 50 cases of functional constipation in children. Chin Pharmaceuticals. 2014;23(09):61-2. [In Chinese].

33.Zhang WS. Clinical efficacy evaluation of combined bacillus subtilis and enterococcus faecium granules with multivitamines (live) in the treatment of functional constipation in children. J Chin Pres Drug. 2016;14(07):61-2. [In Chinese].

34.Zhang XJ, Yuan TT, Xing FX. Study on the clinical effect of Medilac-Vita in the treatment of constipation in children. Chin J of Mod Drug Appl. 2017;11(10):111-2. [In Chinese].

35.Gulati R, Komuravelly A, Leb S, Mhanna MJ, Ghori A, Leon J, et al. Usefulness of Assessment of Stool Form by the Modified Bristol Stool Form Scale in Primary Care Pediatrics. Pediatr Gastroenterol Hepatol Nutr. 2018;21(2):93- 
100.

36.Jozala DR, Oliveira ISF, Ortolan EVP, Oliveira Junior WE, Comes GT, Cassettari VMG, et al. Brazilian Portuguese translation, cross-cultural adaptation and reproducibility assessment of the modified Bristol Stool Form Scale for children. J Pediatr (Rio J). 2019;95(3):321-7.

37.Sun ZQ. Medical Statistics. Beijing: People's Medical Publishing House; 2010.[In Chinese].

38.Lu T, Zhang H, Yin L, Cai J, Li M, Dai L, et al. Chinese pediatric Tuina on children with acute diarrhea: study protocol for a randomized sham-controlled trial. Trials. 2019;20(1):689.

39.Liu Z, Liu J, Zhao Y, Cai Y, He L, Xu H, et al. The efficacy and safety study of electro-acupuncture for severe chronic functional constipation: study protocol for a multicenter, randomized, controlled trial. Trials. 2013;14:176.

40.Xu X, Zheng C, Zhang M, Wang W, Huang G. A randomized controlled trial of acupuncture to treat functional constipation: design and protocol. BMC Complement Altern Med. 2014;14:423.

41.Yang S, Chen J, Guo Y, Teng Y, Liu T, Ying R, et al. Comparison of Taiji and aerobic exercise for functional constipation: study protocol for a randomised controlled neuroimaging trial. BMJ Open. 2019;9(8):e031089.

42.Yin T, He Z, Ma P, Hou L, Chen L, Xie K, et al. Effect and cerebral mechanism of acupuncture treatment for functional constipation: study protocol for a randomized controlled clinical trial. Trials. 2019;20(1):283.

\section{Figures}




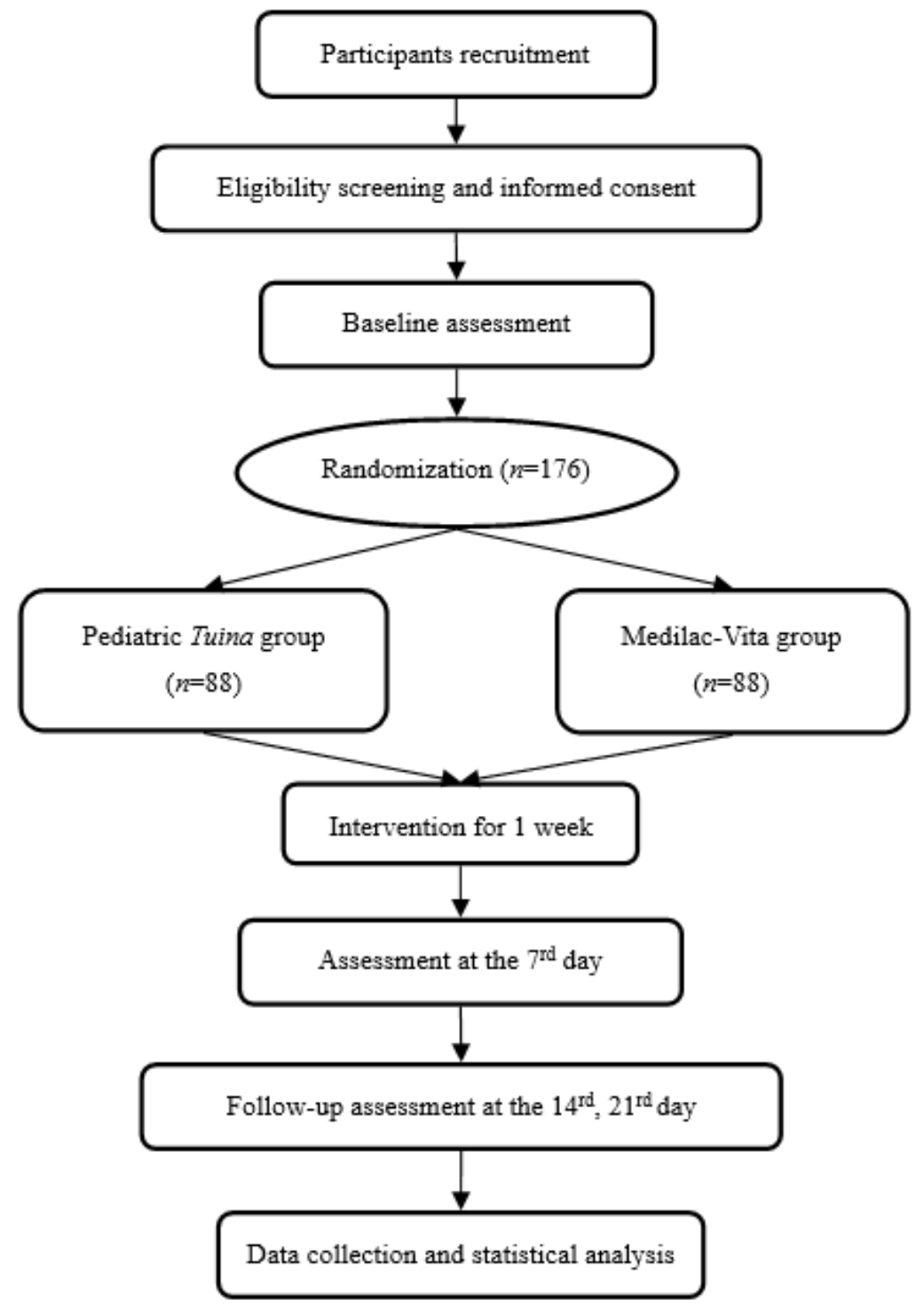

Figure 1

Flowchart of study design
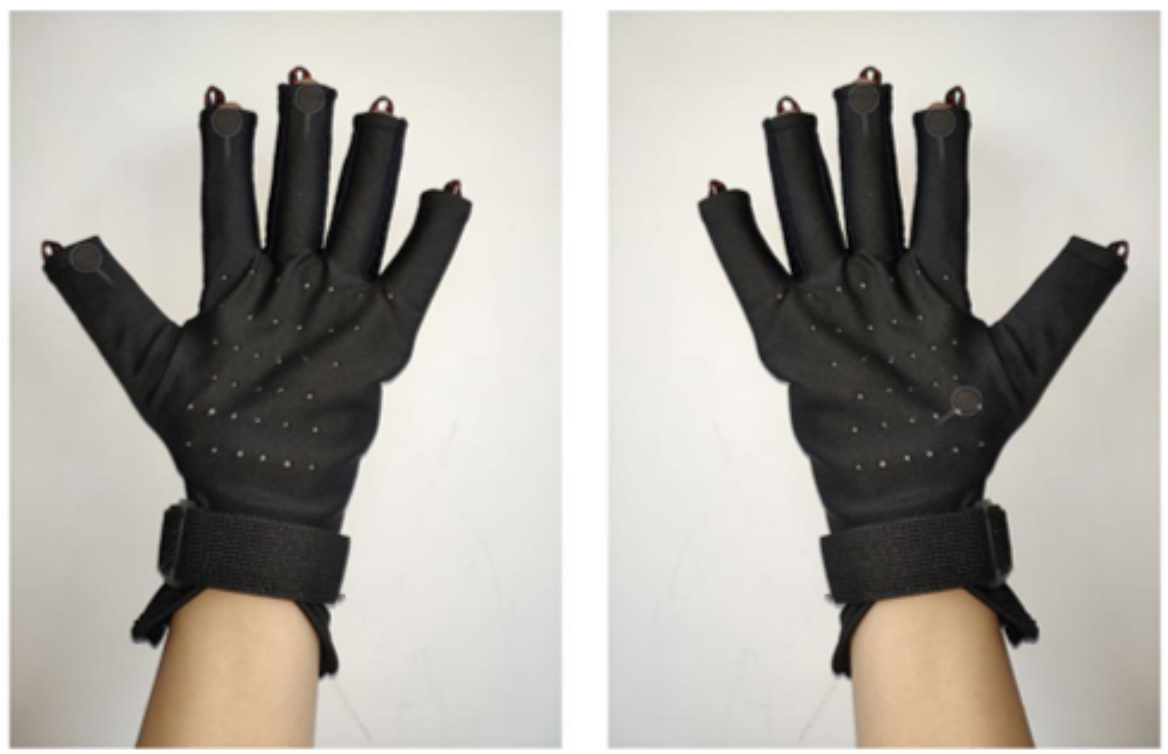
Figure 2

Electronic test glove

\section{Supplementary Files}

This is a list of supplementary files associated with this preprint. Click to download.

- Add1SPIRIT2013Checklist.doc

- Add2DemonstrationvideoofpediatricTuina.rar

- Add3DemonstrationofElectronicTestGlove.mp4

- Add4InstructionofMedilacVita.docx 